\title{
STEADY STATE VIBRATION OF THE PERIODICALLY FORCED AND DAMPED PURE NONLINEAR TWO-DEGREES-OF-FREEDOM OSCILLATOR
}

\author{
Livija Cveticanin \\ University of Novi Sad, Faculty of Technical Sciences, Novi Sad, Serbia, and \\ Óbuda University, Doctoral School of Safety and Security Sciences, Budapest, Hungary \\ Miodrag ZuKovic \\ University of Novi Sad, Faculty of Technical Sciences, Novi Sad, Serbia \\ Dragan CVETICANin \\ Remming, Novi Sad, Serbia \\ e-mail: dragan.cveticanin@remming.co.rs
}

\begin{abstract}
In the paper, a pure nonlinear and damped two-mass oscillator excited with a periodical force is considered. The oscillator is modelled with a system of two coupled second order nonlinear and non-homogenous equations. Using the model, two problems are investigated: one, identification of the excitation force for the known vibrating response of the system, and the second, determination of vibrations of the system excited with the known periodical force. Using the steady-state motion of the nonlinear oscillator, a method for identification of the excitation force is developed. For the pure nonlinear oscillator, it is obtained that the forcing function has the form of the Ateb function. However, if the excitation force is known, the procedure for computing the steady-state vibration of the system is introduced. The solution corresponds to steady-state vibrations of the free oscillator, but the amplitude and phase are assumed to be time variable. The averaged solutions are obtained for the pure nonlinear oscillator with an additional linear elastic force and for the van der Pol oscillator. Analytically obtained solutions are compared with numerical ones. They are in good agreement.
\end{abstract}

Keywords: non-harmonic excitation, force identification, steady-state motion, van der Pol oscillator, Duffing oscillator

\section{Introduction}

Recently, there are many investigations in nonlinear damped systems with periodic (non-harmonic) excitations. Thus, in 2017 more than 500 papers were published where this model or its application was considered (see for example Scopus site). The huge interest in the model is due to the fact that it describes phenomena in many machines and devices but also structures and microelectromechanical (MEMS) and other systems where the excitation is non-harmonic. For example, the influence of environment on the working properties of tools and gear systems of mining machines for coal cutting (Jiang et al., 2016), ground cutting machines, but also in wind turbine geared systems (Wei et al., 2016), is a multifrequency function. Usually, vibration caused by this type of excitation is harmful and causes damage. However, the multi-harmonic excitation in MEMS mixer-filters (Ramini et al., 2016) gives a positive effect in down-conversion and filtering frequencies from radio frequencies to intermediate frequency signals. Vibration of clamped-clamped microbeams in MEMS with periodic excitations are theoretically (Jaber et al., 2016a,b) and experimentally (Ibrahim et al., 2017) investigated. It is shown that there is a benefit of multi-frequency excitations in conversion of the signal in a wide frequency band. The 
periodic and not-harmonic excitation is applied in micro-mirror actuators (Ilyas et al., 2015), too. For all of the mentioned investigations, it is common that the periodic excitation is known and the response of the oscillator is determined mainly experimentally.

On the other hand, there are some results obtained in the identification of periodic excitations based on the response of nonlinear damped or undamped oscillatory systems. In the paper of Jang et al. (2011), a method was developed for identification of the external non-harmonic loading acting on an oscillator with nonlinear restoring and damping. The response of the oscillator was measured and the obtained data were applied for computing the excitation function. In the papers of Hsu (1960), Rakaric et al. (2017) and also in Vakakis and Blanchard (2018), identification of the excitation was based on the steady state motion of the nonlinear and undamped free oscillator. The procedure was limited to one-degree-of freedom oscillators.

In this paper, two problems are considered: 1) periodic forcing identification in a two-degree-of-freedom nonlinear damped oscillator based on the steady state motion of the corresponding free oscillator, and 2) for the known periodic excitation, the response of the nonlinear damped oscillator is obtained. The dimensionless mathematical model of the oscillator is

$$
\begin{aligned}
& \ddot{x}+c(x-y)|x-y|^{\alpha-1}+\lambda(\dot{x}-\dot{y})|\dot{x}-\dot{y}|^{\beta-1}=F(t)+\varepsilon f(x-y, \dot{x}-\dot{y}) \\
& \ddot{y}-c(x-y)|x-y|^{\alpha-1}-\lambda(\dot{x}-\dot{y})|\dot{x}-\dot{y}|^{\beta-1}=-\varepsilon f(x-y, \dot{x}-\dot{y})
\end{aligned}
$$

where $c$ is the stiffness coefficient, $\lambda$ is the damping coefficient, $\alpha, \beta>0, \alpha, \beta \in R$ are orders on nonlinearity (integer or non-integer values) and $F(t)$ is the periodic excitation force. $\varepsilon$ is a small positive parameter $\varepsilon \ll 1$ which gives the small $\varepsilon f$ function. System (1.1) has two coupled strong nonlinear equations with a time-periodic excitation. Already, some special cases of the oscillator are investigated. Thus, free vibration of the nonlinear two-degree-of-freedom oscillator, where the solution is assumed in the form of the Ateb function, is considered (Cveticanin, 2015). In the papers of Kovacic and Zukovic (2017) and of Cveticanin and Zukovic (2017), a two-degree-of-freedom undamped Duffing oscillator with cubic nonlinearity excited with the periodical force of the Jacobi elliptic type was investigated. The steady state amplitude-frequency relations were computed.

Model (1.1) represent a general type of a pure nonlinear oscillator. An analytical method for identification of the excitation force for system (1.1) based on the assumption that the response of the system corresponds to free oscillations is developed. Besides, for different time-periodic excitations, steady-state solutions are obtained. In the paper, the perturbed motion of the oscillator around the steady state is computed. The known asymptotic averaging method for strong nonlinear perturbed differential equations (Cveticanin, 2018b) is adopted for solving Eqs. (1.1). The method is based on the time variable amplitude and phase. The suggested procedure is applied for an approximate solution of the oscillator with an additional linear elastic restitution force and for the van der Pol oscillator. The obtained analytical solutions are compared with numerical ones. They show a good agreement.

\section{Excitation force identification}

By adding and subtracting equations in (1.1), the transformed equations are

$$
\ddot{z}+2 c z|z|^{\alpha-1}+2 \lambda \dot{z}|\dot{z}|^{\beta-1}=F(t)+2 \varepsilon f(z, \dot{z}) \quad \ddot{s}=F(t)
$$

where

$$
z=x-y, s=x+y
$$


Equation $(2.1)_{1}$ is an uncoupled non-homogenous second order strong nonlinear equation, while in Eq. $(2.1)_{2}$ the variable $s$ is the second derivative of the excitation force $F(t)$. Relation $(2.1)_{2}$ does not depend on the perturbation force $\varepsilon f$. It depends only on the external excitation.

First, uncoupled equation $(2.1)_{1}$ has to be considered. Equation $(2.1)_{1}$ represents an oscillator whose motion is perturbed with a small function $\varepsilon f$. The corresponding unperturbed equation is

$$
\ddot{z}+2 c z|z|^{\alpha-1}+2 \lambda \dot{z}|\dot{z}|^{\beta-1}=F(t)
$$

Unfortunately, in Eq. (2.3), the excitation force $F(t)$ is unknown. Our aim is to identify this periodic function. Vakakis and Blanchard (2018) developed an identification method for the special case of this equation when $\alpha=3$ and $\beta=1$. They applied the steady state solution in the form of the Jacobi elliptic function. Using the suggested procedure, the force identification in Eq. (2.3) is done.

We decompose the excitation $F(t)$ in undamped $F_{u}(t)$ and damped $F_{d}(t)$ components $\left(F(t)=F_{u}(t)+F_{d}(t)\right)$ and compute each component separately by satisfying the following problems

$$
\ddot{z}+2 c z|z|^{\alpha-1}=F_{u}(t) \quad 2 \lambda \dot{z}|\dot{z}|^{\beta-1}=F_{d}(t)
$$

Equation $(2.4)_{1}$ corresponds to the undamped pure nonlinear oscillator. Assuming that the vibration is steady-state, undamped subproblem $(2.4)_{1}$ requires the force to be proportional to $z$, i.e.

$$
F_{u}=-U_{1} z|z|^{\alpha-1}
$$

where $U_{1}$ is an unknown value. Substituting Eq. (2.5) into Eq. (2.4) $)_{1}$ one obtains

$$
\ddot{z}+\left(2 c+U_{1}\right) z|z|^{\alpha-1}=0
$$

The exact solution to Eq. (2.6) exists (see Cveticanin, 2018b and Appendix A), and it is

$$
z=\operatorname{Aca}(\alpha, 1, \Omega t)
$$

with

$$
\Omega^{2}=\left(2 c+U_{1}\right) \frac{\alpha+1}{2} A^{\alpha-1}
$$

where $c a=c a(\alpha, 1, \Omega t)$ is the cosine Ateb periodic function (Rosenberg, 1966), $\Omega$ is the frequency of the $c a$ function and $A$ is the amplitude response. The period of the Ateb function is

$$
T=\frac{2 \Pi}{\Omega} \quad \Pi=B\left(\frac{1}{\alpha+1}, \frac{1}{2}\right)
$$

where $B$ is the beta function. Thereby, steady state solution (2.7) is a periodic time function with frequency (2.8) dependent on the amplitude $A$. Substituting solution (2.7) into Eq. (2.4) yields

$$
F_{u}=-U_{1} A c a|A c a|^{\alpha-1}
$$

For the exact steady-state response $z$, we compute the periodic excitation of the oscillator that is required to satisfy Eq. (2.4) $)_{2}$. Namely, using the derivative of Eqs. (2.7) and (2.8), we have

$$
\dot{z}=-A \Omega \frac{2}{\alpha+1} s a(1, \alpha, \Omega t)
$$


where $s a=s a(1, \alpha, \Omega t)$ is the sine Ateb function (Rosenberg, 1966). Applying Eq. (2.11) in Eq. $(2.4)_{2}$, we obtain

$$
F_{d}=-2 \lambda A \Omega \frac{2}{\alpha+1} s a\left|A \Omega \frac{2}{\alpha+1} s a\right|^{\beta-1}
$$

Combining Eq. (2.10) and Eq. (2.12), the periodic force is written in the form

$$
F(t)=F_{u}+F_{d}=P_{1} c a|c a|^{\alpha-1}+P_{2} s a|s a|^{\beta-1}
$$

where

$$
P_{1}=-U_{1} A^{\alpha} \quad P_{2}=-2 A^{\beta} \lambda \Omega^{\beta}\left(\frac{\alpha+1}{2}\right)^{\beta}
$$

According to Eq. (2.8), we have

$$
U_{1}=\Omega^{2} \frac{1}{A^{\alpha-1}} \frac{2}{\alpha+1}-2 c
$$

Applying Eq. (2.13), we obtain the excitation force

$$
F(t)=\left(\Omega^{2} \frac{2 A}{\alpha+1}-2 c A^{\alpha}\right) c a|c a|^{\alpha-1}+2 A^{\beta} \lambda \Omega^{\beta}\left(\frac{\alpha+1}{2}\right)^{\beta} s a|s a|^{\beta-1}
$$

Force (2.16) has two terms. For both of them, it is common that they have the same frequency $\Omega$.

\section{Exact nonlinear resonance}

Using the previous results, let us define the amplitude-frequency relation. Transforming expressions (2.14) into

$$
\frac{P_{2}}{P_{1}}=\frac{2 \lambda \Omega^{\beta}\left(\frac{2}{\alpha+1}\right)^{\beta}}{U_{1} A^{\alpha-\beta}}
$$

and using Eq. (2.15), we obtain the steady state response $A-\Omega$ for various ratios of $P_{2} / P_{1}$

$$
2 \lambda \Omega^{\beta}\left(\frac{2}{\alpha+1}\right)^{\beta} A^{\beta-1}-\frac{P_{2}}{P_{1}}\left(\Omega^{2} \frac{2}{\alpha+1}-2 c A^{\alpha-1}\right)=0
$$

Introducing the relation between the frequencies $\Omega$ and $\omega$ of the Ateb function and of the oscillation, respectively

$$
\omega=\frac{\pi}{\Pi} \Omega=\frac{\pi}{B\left(\frac{1}{\alpha+1}, \frac{1}{2}\right)} \Omega
$$

the amplitude-frequency expression follows

$$
2 \lambda\left(\frac{\Pi}{\pi}\right)^{\beta} \omega^{\beta}\left(\frac{2}{\alpha+1}\right)^{\beta} A^{\beta-1}-\frac{P_{2}}{P_{1}}\left[\left(\frac{\Pi}{\pi}\right)^{2} \omega^{2} \frac{2}{\alpha+1}-2 c A^{\alpha-1}\right]=0
$$

Relation (3.4) is an exact nonlinear resonance relation, i.e. the exact nonlinear resonance curve for the forced and damped system (2.3). In Fig. $1, A-\omega$ diagrams for $P_{2} / P_{1}=-1, \beta=1$ and various values of $\alpha$ are plotted. 


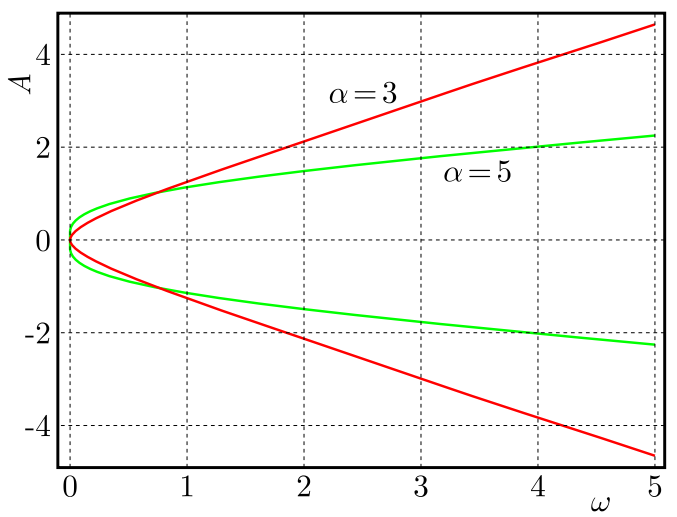

Fig. 1. $A-\omega$ diagrams for $\alpha=3$ and $\alpha=5$

From Fig. 1, it is seen that by increasing the frequency of vibration, the amplitude of vibration increases, too. The velocity of the amplitude increase is faster for smaller orders of the nonlinearity $\alpha$.

For the special case when the damping is linear and $\beta=1$, the amplitude-frequency relation (3.4) simplifies into

$$
\omega=\lambda \frac{\pi}{\Pi}\left(\frac{\alpha+1}{2}\right)^{2} \frac{P_{1}}{P_{2}} \pm \sqrt{\left[\lambda \frac{\pi}{\Pi}\left(\frac{\alpha+1}{2}\right)^{2} \frac{P_{1}}{P_{2}}\right]^{2}+2 c A^{\alpha-1} \frac{\alpha+1}{2}\left(\frac{\pi}{\Pi}\right)^{2}}
$$

We recover the expression for free oscillation of the undamped and unforced truly nonlinear oscillator as given in Cveticanin (2018a).

\section{Exact solution of the unperturbed nonlinear oscillator}

Using Eqs. (2.1) and the previous results, the oscillator excited with periodical force (2.13) yields

$$
\ddot{z}+2 c z|z|^{\alpha-1}+2 \lambda \dot{z}|\dot{z}|^{\beta-1}=P_{1} c a|c a|^{\alpha-1}+P_{2} s a|s a|^{\beta-1} \quad \ddot{S}=P_{1} c a|c a|^{\alpha-1}+P_{2} s a|s a|^{\beta-1}
$$

In Fig. 2, diagrams of excitation force (2.13) for parameters $\Omega=1, P_{1}=1$ and $P_{2}=-1$ and various values of $\alpha$ and $\beta$ are plotted. The values in the excitation force and in the equation are assumed to be non-dimensional.

(a)

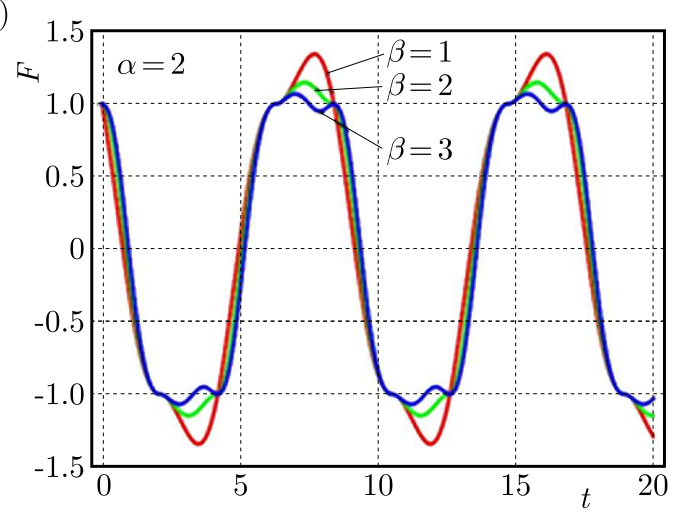

(b)

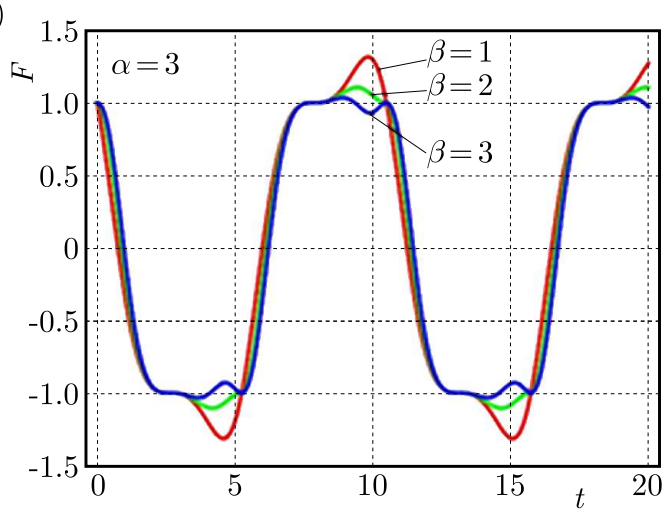

Fig. 2. Excitation force $F(t)$ for $\beta=1,2,3$ and: (a) $\alpha=2$, (b) $\alpha=3$

From the figure, it is obvious that the excitation force is periodical. The period of vibration depends on $\alpha$ and is independent of $\beta$. The period is longer for higher values of $\alpha$. The damping 
coefficient has influence on the amplitude of the excitation force: the higher the values of the damping coefficient, the smaller the amplitude of the force.

For arbitrary parameters of the excitation $P_{1}$ and $P_{2}$, the exact steady state solution to Eqs. (4.1) is (2.7), if relations (2.14) and (2.15) are satisfied. The steady state amplitude of vibration and frequency of the excitation function are

$$
2 \lambda\left(\frac{2}{\alpha+1}\right)^{\beta} \Omega_{S}^{\beta} A_{S}^{\beta}+P_{2}=0 \quad \Omega_{S}=\sqrt{\left(2 c A_{S}^{\alpha-1}-\frac{P_{1}}{A_{S}}\right) \frac{\alpha+1}{2}}
$$

In Tables $1 \mathrm{a}, 1 \mathrm{~b}$ and $1 \mathrm{c}$, values of $A_{S}, \Omega_{S}$ and frequency of vibration $\omega$ (Eq. (3.3)), respectively, for $c=1, \lambda=1, P_{1}=1$ and $P_{2}=-1$ are presented.

Table 1a. $A_{S}$ for various values of $\alpha$ and $\beta$

\begin{tabular}{|c|c|c|c|}
\hline$A_{S}$ & $\alpha=1$ & $\alpha=2$ & $\alpha=3$ \\
\hline \hline$\beta=1$ & 0.68301 & 0.84902 & 0.91754 \\
\hline$\beta=2$ & 0.80902 & 0.94664 & 1.00000 \\
\hline$\beta=3$ & 0.86439 & 0.98884 & 1.03498 \\
\hline
\end{tabular}

Table 1b. $\Omega_{S}$ for various values of $\alpha$ and $\beta$

\begin{tabular}{|c|c|c|c|}
\hline$\Omega_{S}$ & $\alpha=1$ & $\alpha=2$ & $\alpha=3$ \\
\hline \hline$\beta=1$ & 0.73205 & 0.88336 & 1.08987 \\
\hline$\beta=2$ & 0.87403 & 1.12040 & 1.41414 \\
\hline$\beta=3$ & 0.91882 & 1.20401 & 1.53274 \\
\hline
\end{tabular}

Table 1c. $\omega$ for various values of $\alpha$ and $\beta$

\begin{tabular}{|c|c|c|c|}
\hline$\omega$ & $\alpha=1$ & $\alpha=2$ & $\alpha=3$ \\
\hline \hline$\beta=1$ & 0.73025 & 0.65973 & 0.65291 \\
\hline$\beta=2$ & 0.80902 & 0.83678 & 0.84721 \\
\hline$\beta=3$ & 0.87403 & 0.89918 & 0.91882 \\
\hline
\end{tabular}

Analysing the results presented in Table 1a, it is concluded that for the case when the order of the elastic term $\alpha$ is constant, the amplitude of vibration is higher for higher values of the order of the damping $\beta$. If the order $\beta$ of damping is constant, the amplitude of vibration grows by the increasing of $\alpha$.

The frequency of the function $\Omega_{S}$ and of vibration $\omega$ is also increasing with $\beta$ (see Tables $1 \mathrm{~b}$ and 1c). However, the frequency $\omega$ of vibration decreases with an increase in $\alpha$ up to the minimum value, and after that it increases also with $\alpha$. The position of the minimal frequency is moving toward higher values of $\alpha$ for smaller values of $\beta$.

Using the values $A_{S}$ and $\Omega_{S}$ in Tables $1 \mathrm{a}$ and $1 \mathrm{~b}$ and substituting into Eq. (2.7), the exact solution to Eq. $(4.1)_{1}$ is obtained.

\subsection{Oscillator with linear damping}

For oscillator $(4.1)_{1}$ with linear damping, which is excited with the force

$$
F(t)=P_{1} c a|c a|^{\alpha-1}+P_{2} s a
$$

the exact solution is

$$
z=A_{S} c a\left(\alpha, 1, \Omega_{S}, t\right)
$$


For excitation force (4.2), equation $(4.1)_{2}$ is

$$
\ddot{s}=P_{1} c a|c a|^{\alpha-1}+P_{2} s a
$$

Integrating Eq. (4.4) for the initial conditions $s(0)=0$ and $\dot{s}(0)=0$, we have

$$
\dot{s}=\frac{1}{\Omega} P_{1} s a-\frac{\alpha+1}{2 \Omega} P_{2}(c a-1) \quad S=\frac{\alpha+1}{2 \Omega^{2}}\left(P_{1}+P_{2} t\right)-\frac{P_{1}}{\Omega^{2}} \frac{\alpha+1}{2} c a-\frac{\alpha+1}{2 \Omega} P_{2} I
$$

where the integral $I$ is given in Appendix B. Finally, substituting Eq. (4.3) and Eq. (4.5) 2 into Eq. (2.2), the motion of masses is

$$
\begin{aligned}
& x=\frac{\alpha+1}{4 \Omega^{2}}\left(P_{1}+P_{2} t\right)+\frac{2 \Omega^{2} A_{S}-P_{1}(\alpha+1)}{4 \Omega_{S}^{2}} c a-\frac{\alpha+1}{4 \Omega_{S}} P_{2} I \\
& y=\frac{\alpha+1}{4 \Omega^{2}}\left(P_{1}+P_{2} t\right)-\frac{2 \Omega^{2} A_{S}+P_{1}(\alpha+1)}{4 \Omega_{S}^{2}} c a-\frac{\alpha+1}{4 \Omega_{S}} P_{2} I
\end{aligned}
$$

It can be seen that the motion of both masses is a sum of translational and oscillatory motion. Vibrations of the masses are around the trajectory

$$
x_{t}=y_{t}=\frac{\alpha+1}{4 \Omega^{2}}\left(P_{1}+P_{2} t\right)
$$

and are expressed as

$$
\begin{aligned}
& x_{\nu}=\frac{2 \Omega_{S}^{2} A_{S}-P_{1}(\alpha+1)}{4 \Omega_{S}^{2}} c a-\frac{\alpha+1}{4 \Omega_{S}^{2}} P_{2} I \\
& y_{\nu}=-\frac{2 \Omega_{S}^{2} A_{S}+P_{1}(\alpha+1)}{4 \Omega_{S}^{2}} c a-\frac{\alpha+1}{4 \Omega_{S}^{2}} P_{2} I
\end{aligned}
$$

\subsubsection{Duffing oscillator}

Let us consider the two-degree-of-freedom Duffing oscillator $(\alpha=3)$ with linear damping $(\beta=1)$ and parameters $P_{1}=1, P_{2}=-1, c=1, \lambda=1$

$$
\ddot{z}+2 z^{3}+2 \dot{z}=c a^{3}-s a \quad \ddot{s}=c a^{3}-s a
$$

For the initial conditions $z(0)=0$ and $\dot{z}(0)=0$, and using the data from Table 1 , the exact analytical solution to Eq. $(4.8)_{1}$ which describes relative motion of the masses is

$$
z=0.91754 c a(3,1,1.08987 t)
$$

Using relations (4.5), oscillatory motion of the masses is obtained

$$
x_{\nu}=-0.38311 c a+0.84188 I_{3} \quad y_{\nu}=-1.3007 c a+0.84188 I_{3}
$$

where $I_{3}=\int_{0}^{t} c a(3,1,1.08987 t) d t$ is computed numerically according to Appendix B. In Fig. 3a, the $z$ - $t$ diagram for (4.9), and in Fig. 3b diagrams for (4.10) of oscillatory motion for both masses are plotted. It is seen that the period of vibration for both masses is equal while amplitudes of vibration are different. Mass 1 has the amplitude of vibration $A_{1}=0.38311$ while mass 2 the amplitude $A_{2}=1.3008$. 
(a)

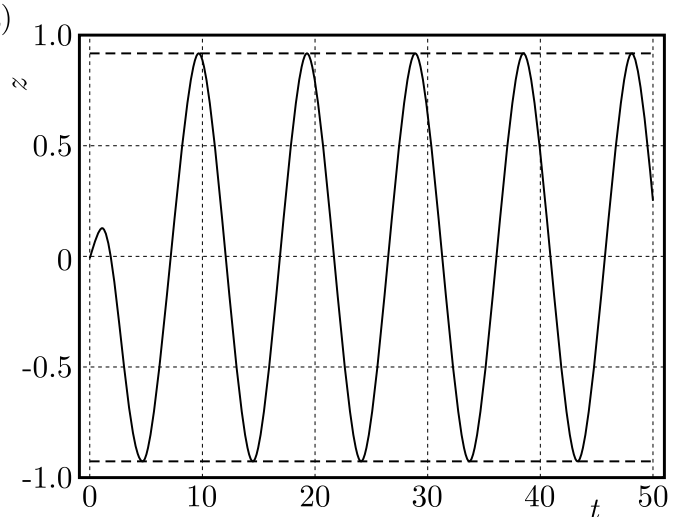

(b)

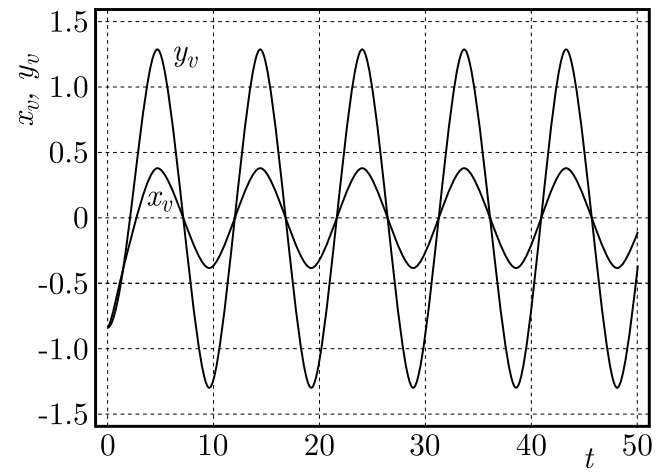

Fig. 3. Amplitude-frequency diagrams for Duffing oscillator: (a) $z$ - $t$ diagram, Eq. (4.9), (b) $x_{\nu}(t)$ and $v_{\nu}(t)$ diagrams, Eq. (4.10), for mass 1 and mass 2, respectively

\subsubsection{Oscillator with quadratic nonlinearity}

For the oscillator with quadratic nonlinearity and linear damping, where the parameters are $P_{1}=1, P_{2}=-1, c=1, \lambda=1$, the equations of motion are

$$
\ddot{z}+2 z|z|+2 \dot{z}=c a|c a|-s a \quad \ddot{s}=c a|c a|-s a
$$

For the initial conditions $z(0)=0$ and $\dot{z}(0)=0$, the exact analytical solution to Eq. $(4.7)_{1}$ is

$$
z=0.8490244 c a(2,1,0.88337 t)
$$

Using Eqs. (4.7), oscillatory motion of the masses is obtained

$$
x_{\nu}=-0.53660 c a+0.96111 I_{2} \quad y_{\nu}=-1.38562 c a+0.96111 I_{2}
$$

where $I_{2}=\int_{0}^{t} c a(2,1,0.88337 t) d t$. In Fig. $4 \mathrm{a}, z-t$ and in Fig. $4 \mathrm{~b}, x_{\nu^{-}} t$ and $y_{\nu^{-}} t$ oscillatory diagrams are plotted.

Analysing pure oscillatory motion of masses 1 and 2 (see Fig. 4b), it is evident that the period of vibration for both masses is equal while mass 1 has the amplitude $A_{1}=0.53660$ and mass 2 the amplitude $A_{2}=1.38562$.

(a)

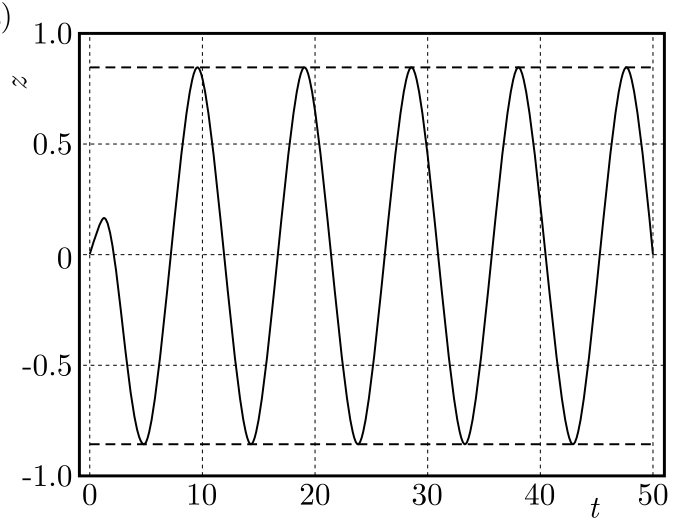

(b)

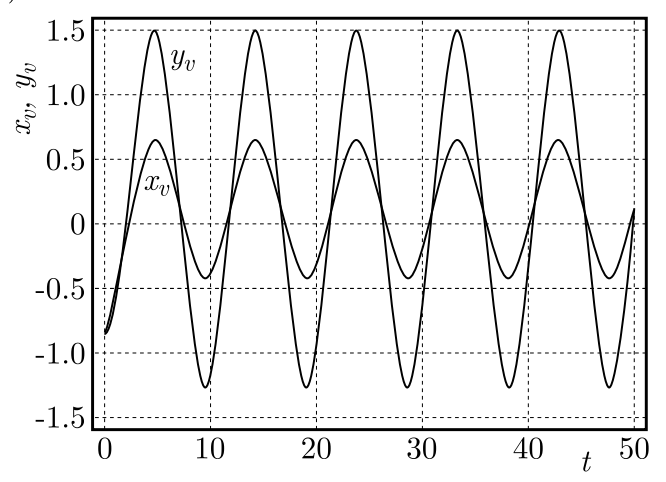

Fig. 4. Amplitude-frequency diagrams for the oscillator with quadratic damping: (a) $z$ - $t$ diagram, Eq. (4.12), (b) $x_{\nu}(t)$ and $y_{\nu}(t)$ diagrams, Eq. (4.13), for mass 1 and mass 2 , respectively

Comparing Fig. 3 and Fig. 4, we see that the amplitude of relative oscillatory motion $z$ is higher for a higher order of the nonlinearity $\alpha$. Besides the amplitudes of vibration of both masses in the system are smaller if the nonlinearity is higher. 


\section{Approximate solution for the perturbed oscillator}

We consider the case when the oscillator is perturbed with a small force. Using model (2.1), it is seen that the perturbation force has an effect on relative motion of the masses. Solving equation (2.1), the influence of perturbation on the relative motion is obtained.

The solving procedure is based on the assumption that the solution of perturbed equation (2.1) is a perturbed version of the exact solution of unperturbed oscillator (2.3)

$$
\ddot{z}+2 c z|z|^{\alpha-1}+2 \lambda \dot{z}|\dot{z}|^{\beta-1}=P_{1} c a|c a|^{\alpha-1}+P_{2} s a|s a|^{\beta-1}
$$

Namely, using Eqs. (2.7) and (2.8) with Eq. (2.15), we assume the solution to $(2.1)_{1}$ in the form

$$
z=A(t) c a(\alpha, 1, \psi(t)) \quad \dot{z}=-A(t) \frac{2}{\alpha+1} \Omega s a(1, \alpha, \psi(t))
$$

where

$$
\dot{\psi}=\Omega+\dot{\theta}(t)
$$

$\Omega$ is according to Eq. (3.2)

$$
K A(t)^{\beta} \Omega^{\beta}=A(t) \Omega^{2} \frac{2}{\alpha+1}-2 c(A(t))^{\alpha}
$$

with

$$
K=2 \lambda \frac{P_{1}}{P_{2}}\left(\frac{2}{\alpha+1}\right)^{\beta}
$$

and $A(t)$ and $\theta(t)$ are unknown time functions. Namely, as equation $(2.1)_{1}$ is the perturbed version of Eq. (2.3), it is supposed that its solution (5.2) has to be the perturbed version of Eqs. (2.7) and (2.8).

Let us rewrite equation $(2.1)_{1}$ in the new variables $A$ and $\theta$. Comparing the time derivative of Eq. (5.2) 1

$$
\dot{z}=-A(t) \frac{2}{\alpha+1} \Omega s a(1, \alpha, \psi(t))+\dot{A}(t) c a(\alpha, 1, \psi(t))-A(t) \frac{2}{\alpha+1} \dot{\theta}(t) s a(1, \alpha, \psi(t))
$$

with Eq. $(5.2)_{2}$, the constraint follows as

$$
\dot{A} c a-A \frac{2}{\alpha+1} \dot{\theta} s a=0
$$

where $A(t)=A, \theta(t)=\theta, \psi(t)=\psi, s a(1, \alpha, \psi(t))$ and $c a(\alpha, 1, \psi(t))=c a$. Substituting Eqs. (5.2) and the time derivative of Eq. $(5.2)_{2}$

$$
\ddot{z}=-\dot{A} \frac{2}{\alpha+1} \Omega s a-A \frac{2}{\alpha+1} \dot{\Omega} s a-A \frac{2}{\alpha+1} \Omega^{2} c a^{\alpha}-A \frac{2}{\alpha+1} \Omega \dot{\theta} c a^{\alpha}
$$

into Eq. $(2.1)_{1}$, and after some modification, we obtain

$$
-(\dot{A} \Omega+A \dot{\Omega}) \frac{2}{\alpha+1} s a-A \frac{2}{\alpha+1} \Omega \dot{\theta} c a^{\alpha}=2 \varepsilon f\left(A c a,-A \frac{2}{\alpha+1} \Omega s a\right)
$$

where $\Omega(A)$. Substituting the time derivative of Eq. (5.4) into Eq. (5.9), it follows

$$
\dot{A} \Phi(A, \Omega) s a+A \frac{2}{\alpha+1} \Omega \dot{\theta} c a^{\alpha}=-2 \varepsilon f\left(A c a,-A \frac{2}{\alpha+1} \Omega s a\right)
$$


where

$$
\Phi(A, \Omega)=\frac{4}{\alpha+1} \frac{\Omega^{2}+c \alpha(\alpha+1) A^{\alpha-1}}{4 \Omega-K \beta(\alpha+1)(A \Omega)^{\beta-1}}
$$

Equations (5.7) and (5.10) with Eq. (5.11) represent the equation of motion of the oscillator with perturbation $(2.1)_{1}$ rewritten into the variables $A$ and $\theta$. Separating the derivatives $\dot{A}$ and $\dot{\theta}$ in Eq. (5.7) and Eq. (5.10), the following relations are obtained

$$
\begin{aligned}
& \dot{A}\left[\Phi(A, \Omega) s a^{2}+\Omega c a^{\alpha+1}\right]=-2 \varepsilon f\left(A c a,-A \frac{2}{\alpha+1} \Omega s a\right) s a \\
& A \dot{\theta} \frac{2}{\alpha+1}\left[\Phi(A, \Omega) s a^{2}+\Omega c a^{\alpha}\right]=-2 \varepsilon f\left(A c a,-A \frac{2}{\alpha+1} \Omega s a\right) c a
\end{aligned}
$$

This is the point the averaging procedure is introduced. Averaging the Ateb functions over period (2.9), it is

$$
\begin{aligned}
& \dot{A}\left\langle\Phi(A, \Omega) s a^{2}+\Omega c a^{\alpha+1}\right\rangle=-2\left\langle\varepsilon f\left(A c a,-A \frac{2}{\alpha+1} \Omega s a\right) s a\right\rangle \\
& A \dot{\theta} \frac{2}{\alpha+1}\left\langle\Phi(A, \Omega) s a^{2}+\Omega c a^{\alpha}\right\rangle=-2\left\langle\varepsilon f\left(A c a,-A \frac{2}{\alpha+1} \Omega s a\right) c a\right\rangle
\end{aligned}
$$

Solution to averaged equations (5.13) gives an approximate solution for $z$. For the special case when

$$
\beta=\frac{2 \alpha}{\alpha+1}
$$

the averaged equations simplify into

$$
\begin{aligned}
& \dot{A} \Omega=-2\left\langle\varepsilon f\left(A c a,-A \frac{2}{\alpha+1} \Omega s a\right) s a\right\rangle \\
& A \Omega \dot{\theta} \frac{2}{\alpha+1}=-2\left\langle\varepsilon f\left(A c a,-A \frac{2}{\alpha+1} \Omega s a\right) c a\right\rangle
\end{aligned}
$$

The order of damping is limited in the interval $\beta \in[1,2)$, while the order of nonlinearity is $\alpha \in[1, \infty)$.

Two special cases are considered: first, when the perturbation depends only on $z$ and second, when it is of van der Pol type.

\subsection{Oscillator perturbed by a small displacement function}

In the case when the perturbation depends only on the relative displacement $z$, the averaged equations (5.13) are

$$
\dot{A} \Omega=-2\langle\varepsilon f(A c a) s a\rangle \quad A \Omega \dot{\theta} \frac{2}{\alpha+1}=-2\langle\varepsilon f(A c a) c a\rangle
$$

After averaging, it is $A=A_{S}=$ const, i.e., the amplitude is equal to the steady state one, whereas $\theta=\omega t$, where

$$
\omega=-\frac{\alpha+1}{A_{S} \Omega_{S}}\left\langle\varepsilon f\left(A_{S} c a\right) c a\right\rangle
$$

Finally, the approximate relative motion of the mass-in-mass oscillator is

$$
Z=A_{S} c a\left(\alpha, 1,\left(\Omega_{S}+\omega\right) t\right)
$$


where the period of vibration is

$$
T=\frac{2 \Pi}{\Omega_{S}+\omega}=\frac{2 B\left(\frac{1}{\alpha+1}, \frac{1}{2}\right)}{\Omega_{S}-\frac{\alpha+1}{A_{S} \Omega_{S}}\left\langle\varepsilon f\left(A_{S} c a\right) c a\right\rangle}
$$

and $A_{S}$ and $\Omega_{S}$ are given in Tables 1a and 1c. The period of vibration is longer for the perturbed than for the unperturbed oscillator.

If the perturbation is by a small linear elastic force and $\varepsilon f=-\varepsilon z$, the period of vibration is

$$
T=\frac{2 B\left(\frac{1}{\alpha+1}, \frac{1}{2}\right)}{\Omega_{S}+\varepsilon \frac{\alpha+1}{\Omega_{S}}\left\langle c a^{2}\right\rangle}
$$

It is obvious that the period of vibration is shorter for the perturbed oscillator than for the unperturbed one: the period is decreasing by the increasing of the perturbation parameter $\varepsilon$.

To prove the accuracy of Eq. (5.19), the analytically obtained period is compared with the numerically obtained one by solving the equation of motion

$$
\ddot{z}+2 z|z|^{\alpha-1}+2 \dot{z}=c a|c a|^{\alpha-1}-s a-2 \varepsilon z
$$

In Table 2, the analytically and numerically calculated periods of vibration for $\varepsilon=0.1$ and various values of $\alpha$ are compared.

Table 2. Periods of vibration $T$ and $T_{N}$ as functions of $\alpha$

\begin{tabular}{|c|c|c|c|}
\hline & $\alpha=2$ & $\alpha=5 / 2$ & $\alpha=3$ \\
\hline \hline$T$ & 9.37856 & 9.51909 & 9.61999 \\
\hline$T_{N}$ & 9.38163 & 9.52003 & 9.62117 \\
\hline
\end{tabular}

It is obvious that the difference between the analytically and numerically calculated periods is negligible.

\subsection{Oscillator of van der Pol type}

If the perturbation is of the van der Pol type is introduced

$$
\varepsilon f(z, \dot{z})=\varepsilon \dot{z}\left(1-z^{2}\right)
$$

the averaged equations (5.15) are transformed into

$$
\dot{A}=\frac{4 \varepsilon A}{\alpha+1}\left(\left\langle s a^{2}\right\rangle-A^{2}\left\langle s a^{2} c a^{2}\right\rangle\right) \quad \dot{\theta}=0
$$

Solving the first order Abel equation (5.22), we obtain

$$
A=\frac{\varepsilon A_{S} \exp \left(\frac{2\left\langle s a^{2}\right\rangle}{\alpha+1} t\right)}{\sqrt{1-\varepsilon^{2} A_{S}^{2} \frac{\left\langle s a^{2} c a^{2}\right\rangle}{\left\langle s a^{2}\right\rangle}\left[1-\exp \left(\frac{4\left\langle s a^{2}\right\rangle}{\alpha+1} t\right)\right]}} \quad \theta=0
$$

Analysing Eq. (5.23), it is seen that for the limit case, when $t \rightarrow \infty$, the amplitude of vibration tends to the steady state value

$$
A=\sqrt{\frac{\left\langle s a^{2}\right\rangle}{\left\langle s a^{2} c a^{2}\right\rangle}}
$$


Thus, the van der Pol oscillator

$$
\ddot{z}+2 z|z|^{\alpha-1}+2 \dot{z}=c a|c a|^{\alpha-1}-s a+2 \varepsilon \dot{z}\left(1-z^{2}\right)
$$

tends to the limit cycle motion with amplitude (5.24).

In the case when the order of nonlinearity is $\alpha=3$, the limit cycle amplitude is $A=0.91875$, and for $\alpha=2$ it is $A=0.850892$. Comparing the values with those in Table 1, it is obvious that the van der Pol perturbation causes an increase in the vibration amplitude in comparison to the unperturbed oscillator.

\section{Conclusions}

In this paper, generalization of two mass two-degree-of-freedom pure nonlinear oscillators with strong damping and a non-harmonic excitation is considered. First, the method for identification of the excitation force in the nonlinear and damped oscillator with two-degrees-of-freedom is developed. The suggested procedure is based on application of the exact steady state solution of the free oscillator obtained analytically. The computed multi-periodic force is simplified into the periodic Ateb function, which is very convenient for mathematical application. The excitation force is the sum of $c a$ and $s a$ functions whose order depends on the nonlinearity and damping orders of the system.

In the paper, the exact nonlinear resonance expression is determined. For the pure nonlinear oscillator, only one stable branch of the amplitude-frequency curve exists. For a higher order of nonlinearity, the bending of the curve is higher.

In the paper, the response of the oscillator excited with a certain periodic force is investigated. Condition (4.1) for the steady state solution which corresponds to motion of the free undamped oscillator is obtained. The exact values of frequency and amplitude parameters of vibration are computed. Analysing the results, it is concluded that for a higher order of nonlinearity and a higher order of damping, the amplitude of vibration is higher. However, the frequency of vibration decreases with the increasing of the order of nonlinearity up to the minimum value, and after that it increases. The position of the minimum frequency is moving toward smaller values of order of the nonlinearity for higher values of damping.

If steady-state motion of the oscillator is perturbed, the amplitude and frequency of oscillations are varying in time. In the paper, a procedure for solving perturbed motion of the strong nonlinear, damped and excited oscillator based on the assumption that for a small perturbation the variation of motion in comparison to the unperturbed one is quite small is developed. Special attention is given to two problems: first, when the perturbation function is a linear function of displacement and second, when the van der Pol damping force acts. For linear perturbation, the amplitude of vibration is not varying, but the period of vibration is shortening. In the case of van der Pol perturbation, the limit cycle motion is obtained. The approximate steady state limit value depends on the order of nonlinearity and it is higher the higher the order of nonlinearity. The obtained analytical results are compared with numerical ones. They are in a good agreement.

The results presented in the paper would be interesting in the design of structures with periodic forcing but steady state oscillatory motion. This type of motion would increase the working life of the system.

In this paper, the integral of the $c a$ Ateb function is introduced for the first time. The integral is obtained analytically, but also numerically. It is concluded that the obtained function is periodical with a period equal to the period of the corresponding $c a$ function. Increasing the parameter $\alpha$, the period of the obtained integral function is longer and the amplitude is higher. 


\section{Acknowledgements}

This article has been based upon work from COST Action DENORMS - CA15125, supported by COST (European Cooperation in Science and Technology). The investigation has been financially supported by the Ministry of Science, Republic of Serbia (Proj. No. ON174028 and No. III41007).

\section{A. Appendix}

The first integral of Eq. (2.6) is

$$
\frac{\dot{z}^{2}}{2}+K z^{\alpha+1}=Q
$$

where

$$
K=\frac{2 c+U_{1}}{\alpha+1}
$$

and $Q=$ const depending on the initial conditions. For the initial condition $z(0)=A=$ const

$$
Q=K A^{\alpha+1}
$$

Introducing a new variable

$$
\nu=\frac{K}{Q} z^{\alpha+1}
$$

and applying some modifications, Eq. (A.1) transforms into

$$
\frac{1}{\alpha+1} \sqrt{\frac{2}{Q}}\left(\frac{Q}{K}\right)^{\frac{1}{\alpha+1}}\left(\frac{1}{2} \int_{0}^{0 \leqslant z \leqslant 1} \frac{d \nu}{\sqrt{1-\nu} \nu^{\alpha /(\alpha+1)}}\right)=t
$$

Using the definition of the incomplete Beta function

$$
\frac{1}{2} B_{\nu}\left(\frac{1}{\alpha+1}, \frac{1}{2}\right)=\frac{1}{2} \int_{0}^{0 \leqslant z \leqslant 1} \frac{d \nu}{\sqrt{1-\nu} \nu^{\alpha /(\alpha+1)}}
$$

Eq. (A.5) becomes

$$
\frac{1}{2} B_{\nu}\left(\frac{1}{\alpha+1}, \frac{1}{2}\right)=t(\alpha+1) \sqrt{\frac{Q}{2}}\left(\frac{K}{Q}\right)^{\frac{1}{\alpha+1}}
$$

Introducing (A.2) and (A.3) into (A.7) and using the inverse incomplete Beta function (see Cveticanin and Pogany, 2012) the solution to (A.1) has the form of the so called cosine Ateb function (Rosenberg, 1966)

$$
z=A c a\left(\alpha, 1, t(\alpha+1) \sqrt{\frac{2 c+U_{1}}{\alpha+1} \frac{A^{\alpha+1}}{2}} A^{-1}\right)
$$

Finally

$$
z=A c a\left(\alpha, 1, t \sqrt{\frac{\left(2 c+U_{1}\right)(\alpha+1)}{2}} A^{\frac{\alpha-1}{2}}\right)
$$


The frequency of the function is

$$
\Omega=\sqrt{\frac{\left.2 c+U_{1}\right)(\alpha+1)}{2}} A^{\frac{\alpha-1}{2}}
$$

Integrating (A.6) in the interval $[0,1]$, the incomplete Beta function transforms into the complete Beta function

$$
\frac{1}{2} B\left(\frac{1}{\alpha+1}, \frac{1}{2}\right)=\frac{1}{2} \int_{0}^{1} \frac{d \nu}{\sqrt{1-\nu} \nu^{\frac{\alpha}{\alpha+1}}}
$$

Substituting (A.11) into (A.5), a quarter of the period $T$ of the Ateb function is obtained

$$
\frac{T}{4}=\frac{1}{2} \frac{1}{\alpha+1} \sqrt{\frac{2}{Q}}\left(\frac{Q}{K}\right)^{\frac{1}{\alpha+1}} B\left(\frac{1}{\alpha+1}, \frac{1}{2}\right)
$$

Using (A.2) and (A.3) and comparing (A.12) and (A.10), it follows

$$
T=\frac{2 \Pi_{\alpha}}{\Omega}
$$

where

$$
\Pi_{\alpha}=B\left(\frac{1}{\alpha+1}, \frac{1}{2}\right)
$$

The function which has a phase difference of (A.9) corresponds to the sine Ateb function

$$
z=A s a\left(\alpha, 1, \frac{\Pi_{\alpha}}{2}+t \sqrt{\frac{\left(2 c+U_{1}\right)(\alpha+1)}{2}} A^{\frac{\alpha-1}{2}}\right)
$$

Let us mention some of the properties of Ateb functions. The relation between $c a$ and $s a$ Ateb functions is

$$
c a^{\alpha+1}(\alpha, 1, z)+s a^{2}(1, \alpha, z)=1
$$

In addition, the first derivatives of $s a$ and $c a$ functions are

$$
\frac{d}{d z} c a(\alpha, 1, z)=-\frac{2}{\alpha+1} s a(1, \alpha, z) \quad \frac{d}{d z} s a(1, \alpha, z)=c a^{\alpha}(\alpha, 1, z)
$$

\section{B. Appendix}

Let us compute the integral

$$
I=\int_{0}^{t} c a(\alpha, 1, t) d t
$$

Using relation (A.16) and introducing a new variable

$$
\nu=1-s a^{2}(1, \alpha, t)
$$




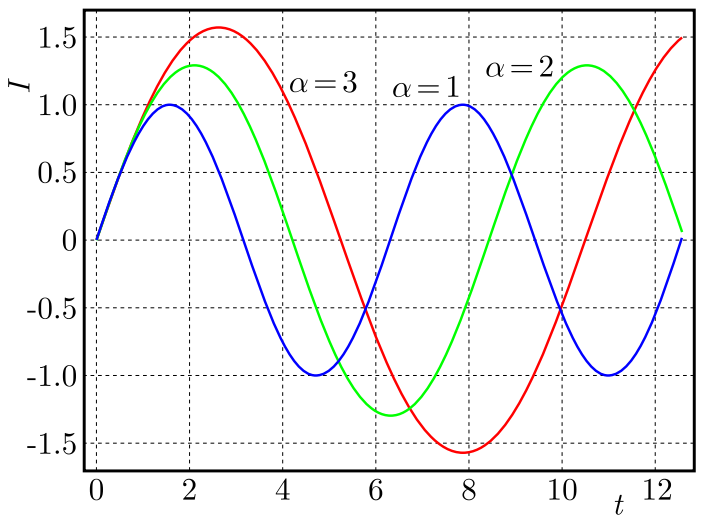

Fig. 5. Integral of the $c a$ function for $\alpha=1$ (blue line), $\alpha=2$ (green line) and $\alpha=3$ (red line)

integral $I$ transforms into

$$
I=-\frac{1}{2} \int_{1}^{\nu} \frac{1}{\sqrt{1-\nu}} \nu^{\frac{1-\alpha}{1+\alpha}} d \nu
$$

Utilizing the definition of the incomplete Beta function $B_{\nu}(a, b)=\int_{\nu} \nu^{a-1}(1-\nu)^{b-1} d \nu$ we have

$$
I=-\frac{1}{2} B_{\nu}\left(\nu, \frac{1}{\alpha+1}, \frac{1}{2}\right)
$$

In Fig. 5, obtained integral (B.4) for various values of $\alpha$ is plotted.

It is seen that the integral of the $c a$ function is a periodical function with a period which is equal to the period of the corresponding $c a$ function $T=2 \Pi_{\alpha}=2 B(1 /(\alpha+1), 1 / 2)$. The amplitude of vibration and period length of integral $I$ is higher for higher values of $\alpha$.

\section{References}

1. Cveticanin L., 2015, A solution procedure based on the Ateb function for a two-degree-of-freedom oscillator, Journal of Sound and Vibration, 346, 298-313

2. Cveticanin L., 2018a, Free vibrations, [In:] Strong Nonlinear Oscillators, Mathematical Engineering, Springer, 9783319588254, 51-117

3. Cveticanin L., 2018b, Pure nonlinear oscillator, [In:] Strong Nonlinear Oscillators, Mathematical Engineering, Springer, 9783319588254, 17-49

4. Cveticanin L., Pogany T., 2012, Oscillator with a sum of non-integer order non-linearities, Journal of Applied Mathematics, 2012, 649050, 20 p.

5. Cveticanin L., Zukovic M., 2017, Negative effective mass in acoustic metamaterial with nonlinear mass-in-mass subsystems, Communications in Nonlinear Science and Numerical Simulation, $\mathbf{5 1}, 89-104$

6. Hsu C., 1960, On the application of elliptic functions in non-linear forced oscillations, Quarterly of Applied Mathematics, 17, 393-407

7. Ibrahim A., Jaber N., Chandran A., Thirupathi M., Younis M., 2017, Dynamics of microbeams under multi-frequewncy excitations, Micromachines MDPI, 32, 8, 1-14

8. Ilyas S., Ramini A., Arevalo A., Younis M.I., 2015, An experimental and theoretical investigation of a micromirror under mixed-frequency excitation, Journal of Microelectromechanical Systems, 24, 1124-1131 
9. Jaber N., Ramini A., Hennawi Q., Younis M.I., 2016a, Multifrequency excitation of a clamped-clamped microbeam: Analytical and experimental investigation, Microsystems and Nanoengineering, 2, 16002

10. Jaber N., Ramini A., Hennawi Q., Younis M.I., Wideband M.I., 2016b, MEMS resonator using multifrequency excitation, Sensors and Actuators A: Physical, 242, 140-145

11. JAng T.S., BAek H., Choi H.S., LeE S.G., 2011, A new method for measuring nonharmonic periodic excitation forces in nonlinear damped systems, Mechanical Systems and Signal Processing, 25, 2219-2228

12. Jiang Y., Zhu H., Li Z., Peng Z., 2016, The nonlinear dynamics response of cracked gear system in a coal cutter taking environmental multi-frequency excitation forces into consideration, Nonlinear Dynamics, 84, 203-222

13. Kovacic I., Zukovic M., 2017, Coupled purely nonlinear oscillators: normal modes and exact solutions for free and forced resonances, Nonlinear Dynamics, 87, 713-726

14. Rakaric Z., Kovacic I., Cartmell M., 2017, On the design of external excitations in order to make nonlinear oscillators respond as free oscillators of the same or different type, International Journal of Non-Linear Mechanics, 94, 323-333

15. Ramini A., Ibrahim A.I., Younis M.I., 2016, Mixed frequency excitation of an electrostatically actuated resonator, Microsystem Technologies, 22, 1967-1974

16. Rosenberg R.M., 1966, On non-linear vibration of systems with many degrees of freedom, Advances in Applied Mechanics, 9, 155-242

17. VAKakis A.F., Blanchard A., 2018, Exact steady states of the periodically forced and damped Duffing oscillator, Journal of Sound and Vibration, 413, 57-65

18. Wei S., Han Q., Peng Z., Chu F., 2016, Dynamic analysis of parametrically excited system under uncertainties and multi-frequency excitations, Mechanical Systems and Signal Processing, 72-73, 762-784 